\title{
Using the first drop of blood for monitoring blood glucose values in critically ill patients: An observational study
}

\author{
Sunita Saini, Sukhpal Kaur ${ }^{1}$, Karobi Das ${ }^{1}$, Vikas Saini ${ }^{2}$
}

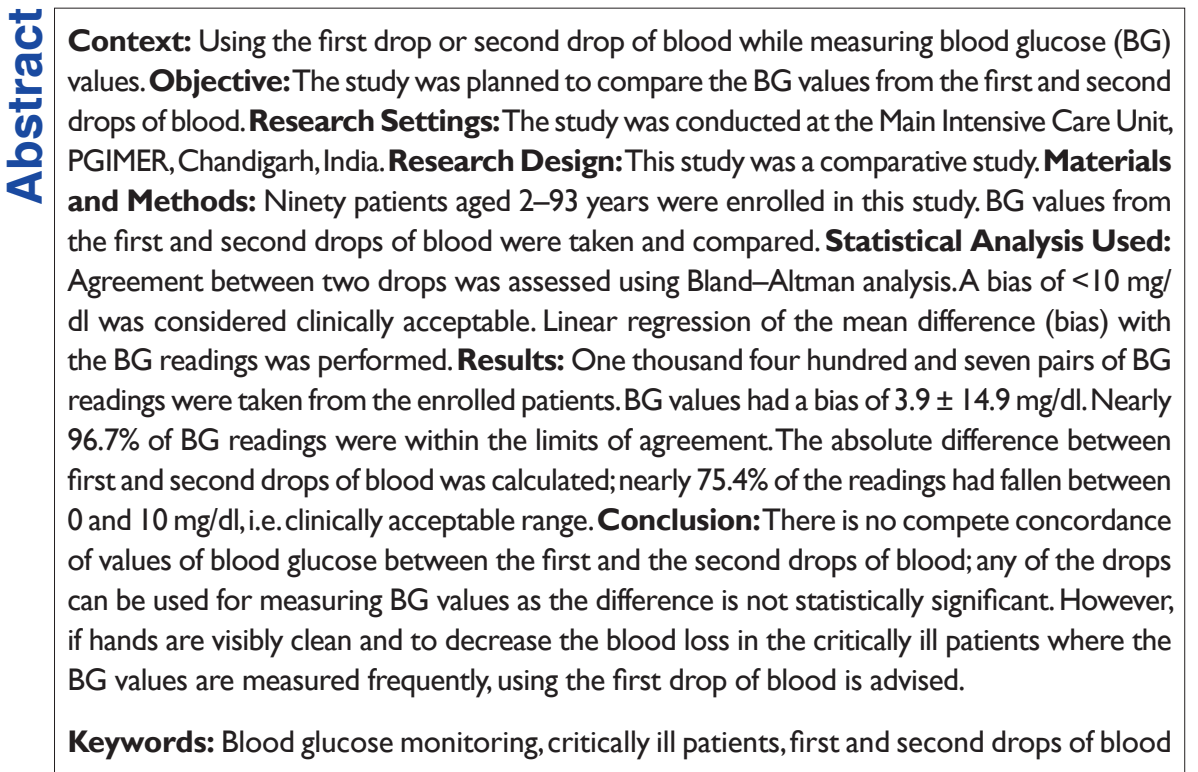

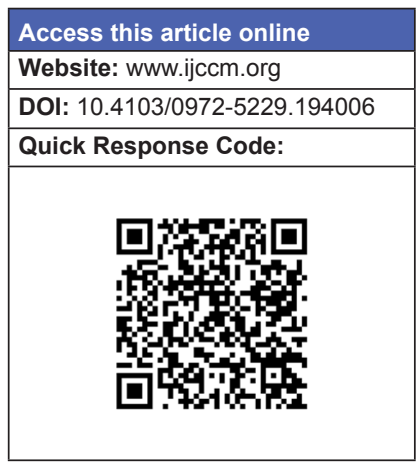

\section{Introduction}

For clinical nurses, especially those working in ICU settings, it is crucial to measure blood glucose values in an accurate, timely and safely manner ${ }^{[1]} \mathrm{BG}$ monitoring would be helpful in safety in terms of falling or rise in BG values, adjusting the insulin dosage, and managing illness. ${ }^{[2]}$ Even brief hypoglycemia can cause profound brain dysfunction while prolonged severe hypoglycemia causes brain death. The Intensive Care Unit (ICU) protocols are to maintain the BG values within the range of $130-180 \mathrm{mg} / \mathrm{dl} .^{[3-5]}$

From:

Department of Nursing, Government Medical College and Hospital, Sector - 32, Chandigarh, ${ }^{1}$ National Institute of Nursing Education, Post Graduate Institute of Medical Education and Research, ${ }^{2}$ Department of Anaesthesia and Critical Care Unit, PGIMER, Chandigarh, India

\section{Correspondence:}

Dr. Sukhpal Kaur, Lecturer, National Institute of Nursing, PGIMER,

Chandigarh, India.

E-mail: sunitasaini840@gmail.com
Effective glucose control in the ICU has been shown to decrease morbidity of conditions and also to decrease mortality. ${ }^{[6]}$ Point-of-care (POC) testing (or near-patient testing), where samples are not sent to the laboratory, is a convenient method commonly used in blood sugar monitoring. ${ }^{[3]}$ POC reduces the laboratory lag time to measure the BG values and is inexpensive, safer, and less invasive $^{[7]}$ than capillary glucose measurements. Many differences in practice exist with regard to use of first or second drop of blood for testing and no consistent

This is an open access article distributed under the terms of the Creative Commons Attribution-NonCommercial-ShareAlike 3.0 License, which allows others to remix, tweak, and build upon the work non-commercially, as long as the author is credited and the new creations are licensed under the identical terms.

For reprints contact: reprints@ @edknow.com

How to cite this article: Saini S, Kaur S, Das K, Saini V. Using the first drop of blood for monitoring blood glucose values in critically ill patients: An observational study. Indian J Crit Care Med 2016;20:658-61. 
guidelines are available for capillary BG testing. ${ }^{[1]}$ The purpose of this study is to evaluate BG differences between first and second drop of capillary blood from same site. In general, used sites are the sides of fingers for sticking. More nerves are present in the center of finger pad, so lancing in this location may cause more pain. If possible, hands should be washed with warm and soapy water, which will, besides cleaning, bring additional blood to the fingers. Rubbing alcohol can, of course, be used but let it dry for 30 s. If fingers are covered with dirt and juice, the best option is to prick the finger and wipe the first drop of blood away with a clean tissue or garment. Test with the second drop of blood, which is less likely to be contaminated. ${ }^{[8]}$

\section{Objectives}

- Developing a protocol for the measurement of BG values

- Comparing the BG values from the first and second drops of blood

- Calculating the bias and limits of agreement for the values of blood sugar level

- Calculating the effect of BG values on bias.

\section{Materials and Methods}

\section{Setting and sampling}

The study was conducted in the ICU of tertiary care teaching hospital. Ethical clearance was taken from Ethics Committee, and consent was taken from patients' attendants. The study sample included ninety patients from whom 1407 pairs of BG readings were taken using comparative research design. Sample was collected from July 16 to September 21, 2014. An inclusion criterion was patients on BG monitoring. Patients with peripheral vascular diseases were excluded from the study.

Two glucometers (code free) were used to take the first and second drops of blood after being numbered 1 and 2 . Code free glucometers are specially designed glucometers as these need not to be calibrated. Meters with no coding technology are available and automatically code to match the test strips - they cannot be miscoded. Meters with no coding technology remove the worry of inaccurate results from a miscode, giving one less thing to think about when managing diabetes and are more accurate as compared with manually coded meters. ${ }^{[9]}$

The glucose strips used were code-free strips. Finger site was cleaned with the spirit swab and was left for letting dry for $30 \mathrm{~s}$. The first drop of blood was taken on a glucose strip after pricking the finger with the needle. Then, the dry swab was used to wipe the finger site and then the second drop of blood was taken on the second glucose strip. Patient was given dry cotton swab to control the bleeding. The readings from both the glucometers were recorded in the BG monitoring chart.

\section{Data collection processing and statistical analysis}

Data collected was analyzed using descriptive, inferential, and Bland-Altman statistics. Analysis was carried out with the help of Microsoft Excel, Gnumeric software, and Statistical Package for Social Science, version 19.0 (Armonk,NY: IBM Corp). The various statistical measures such as frequencies, percentage, mean and standard deviation, and Welch's t-test were employed.

\section{Technical specifications ${ }^{[10]}$ \\ Instrument principle and strip methods}

The SD CodeFree ${ }^{\mathrm{TM}}$ manufactured by SD Biosensor, Inc. C-4th and 5th Floor Digital Empire Building 980-3, Yeongtong-dong, Yeongtong-gu, Suwon-si, Kyonggido, Korea. The SD CodeFree ${ }^{\mathrm{TM}}$ strip is designed with anelectrode that measures glucose levels. Glucose in the blood sample mixes with reagent on the test strip that causes a small electric current. The amount of current that is created depends on how much glucose is in the blood. The SD CodeFree ${ }^{\mathrm{TM}}$ meter measures the current that is created and converts the measurement to the amount of glucose that is in the blood. The BG result is displayed on the meter's LCD display. By touching a drop of blood to the tip of the SD CodeFree ${ }^{\mathrm{TM}}$ test strip, the strip's reaction chamber automatically draws the blood into the strip through capillary action. When the chamber is full, the SD CodeFree $^{\mathrm{TM}}$ meter starts to measure the BG level.

\section{Hematocrit limits ${ }^{[10]}$}

Extremes in hematocrit may affect test results. Hematocrit levels $<20 \%$ may cause falsely high readings. Hematocrit levels $>60 \%$ may cause falsely low readings.

\section{Results $^{[11]}$}

Ninety participants (fifty males and forty females) with the mean age of $34 \pm 18 \mathrm{SD}$ (years) admitted to the ICU on BG monitoring were studied. One thousand four hundred and seven pairs of BG values from the first and second drops of blood were measured. The details of frequency of absolute difference in BG values from the first and second drops of blood are given in Table 1. Table 2 depicts the descriptive statistics of the BG readings from the first and second drops of blood. Table 3 depicts thelimits of agreement. The upper limit of agreement was $33.1 \mathrm{mg} / \mathrm{dl}$ and lower limit of agreement was $-25.3 \mathrm{mg} / \mathrm{dl}$ with the mean difference of $3.9 \pm 14.9$ SD. $96.7 \%$ of the readings were within the limits of agreement. Table 4 shows the mean difference of blood glucose values from the first and second drops 
of blood with the selected variables. The patients who had history of diabetes had mean \pm SD of blood glucose values $167.5 \pm 60.2$ and $163.1 \pm 59.1$ from the first and second drops of blood respectively. The $t$ value is not significant which means that bias was not different in diabetic and non-diabetic patients. Similarly, the patients who were on insulin sliding scale had a mean \pm SD of $183.5 \pm 54.9$ in the first drop of blood and $126.6 \pm 35.7$ in the second drop of blood. The $t$ test is not significant

Table I: Frequency of absolute difference $(\mathrm{mg} / \mathrm{dl})$ in readings of blood glucose values from the first and second drops of blood $(n=1407)$

\begin{tabular}{lc}
\hline Difference in blood glucose values & Frequency of difference, $\boldsymbol{n}(\%)$ \\
\hline $0-10$ & $106 I(75.4)$ \\
$I I-20$ & $244(17.3)$ \\
$2 I-30$ & $52(3.7)$ \\
$3 \mid-40$ & $22(1.6)$ \\
$4 I-50$ & $12(0.9)$ \\
$>5 I$ & $16(1.1)$ \\
\hline
\end{tabular}

Table 2: Blood glucose values $(\mathrm{mg} / \mathrm{dl})$ from the first and second drops of blood $(n=1407)$

\begin{tabular}{lcc}
\hline & First drop & Second drop \\
\hline Mean \pm SD & $\mid 47.7 \pm 5 I .77$ & $\mid 43.80 \pm 5 I .5 \mathrm{I}$ \\
Minimum-maximum blood & $37-403$ & $40-327$ \\
glucose value $(\mathrm{mg} / \mathrm{dl})$ & & \\
\hline SD: Standard deviation & &
\end{tabular}

Table 3: Bias and limits of agreement in the first and second drops of blood $(n=1407)$

\begin{tabular}{lcccc}
\hline Blood glucose & $\begin{array}{c}\text { Upper } \\
\text { limit of } \\
\text { agreement }\end{array}$ & $\begin{array}{c}\text { Lower } \\
\text { limit of } \\
\text { agreement }\end{array}$ & Bias & $\begin{array}{c}\text { Percentage of } \\
\text { readings in limits } \\
\text { of agreement }\end{array}$ \\
\hline $\begin{array}{l}\text { First and second } \\
\text { drops of blood }\end{array}$ & 33.1 & -25.3 & $3.9 \pm 14.9$ & 96.7 \\
\hline
\end{tabular}

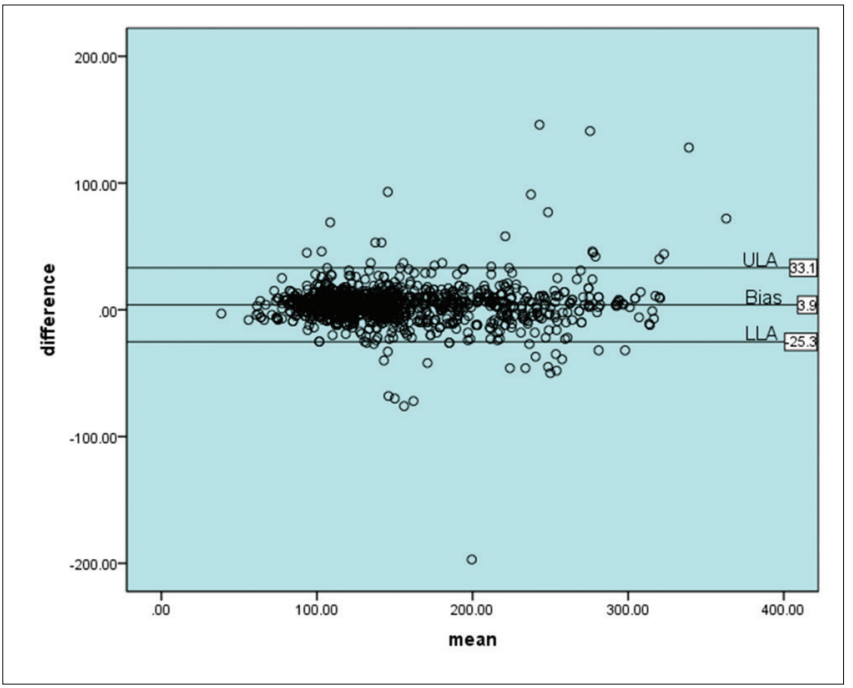

Figure I: Bland-Altman plot of the blood glucose values from the first and second drops of blood with the limits of agreement which means that bias was not different in patients who received insulin or not.

The Bland-Altman plots and the regression analysis of the bias of BG values from the first and second drops of blood are depicted in Figures 1 and 2. Figure 2 depicts that there was a significant increase of bias as the blood sugar values increased $\left(r=0.127, r^{2}=0.016, P<0.001\right)$.

\section{Discussion}

On comparing the results of study by Li 802 groups of BG in 526 patients, no significant difference in the BG levels of the first and second drop of blood and venous blood was found. However, after combining then dividing measurements into six groups according to BG concentration, statistically significant differences between the BG levels obtained from the first drop, second drop, and venous blood in the groups containing BG values $<9.9$ or 20-30 mmol/L. In contrast, there were no significant differences in 10-14.9 or 15-19.9 mmol/L groups. ${ }^{[12]}$

According to a study conducted by Palese et al., a strong correlation emerged between the BG reported in the first and the second drops (Spearman's rho test $\left[r_{\mathrm{s}}\right]=0.979$, $P<0.001$; Pearson $r=0.978, P<0.001$ ). The average BG values obtained from the first and second drops were $184.30 \mathrm{mg} / \mathrm{dL}$ (median, 166) and $187.6 \mathrm{mg} / \mathrm{dL}$ (median, 172), respectively, and thus the second drop showed higher glucose values compared with the first drop. However, BG values of the second drop were not higher in all occasions: Whereas some evaluations reported higher BG values in the second drop capillary sample $(n=123)$, others reported higher values in the first $\operatorname{drop}(n=65)$, and still others reported identical measurements in the first and second drops $(n=7)$. Five outliers were present with a BG difference from -39 to $-53 \mathrm{mg} / \mathrm{dL}$ in the first drop compared with the second drop, and three outliers were present with a BG difference from +46 to $+57 \mathrm{mg} / \mathrm{dL}$ in

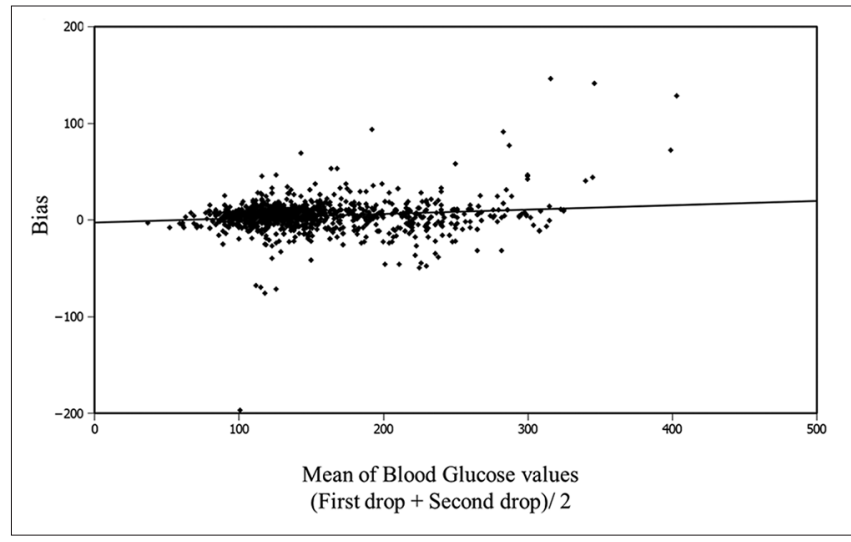

Figure 2: Regression line and scatter plot of the mean difference (bias) and mean 


\begin{tabular}{|c|c|c|c|}
\hline Variables & $n(\%)$ & Mean \pm SD & $t, P$ \\
\hline \multicolumn{4}{|l|}{ Diabetic } \\
\hline Yes & $300(21.3)$ & $189.53 \pm 56.7$ & 1.38 \\
\hline No & II 07 (78.7) & $136.3 \pm 43.9$ & 0.167 \\
\hline \multicolumn{4}{|c|}{ On insulin sliding scale } \\
\hline Yes & $521(37)$ & $183.5 \pm 54.9$ & 1.32, \\
\hline No & $886(63)$ & $126.6 \pm 35.7$ & 0.250 \\
\hline
\end{tabular}

SD: Standard deviation

the first drop compared with the second drop. However, the differences that emerged were not affected by glucose concentration $(P=0.221) \cdot{ }^{[9]}$ This may be because the glucose meter measurements may be subjected to artifacts resulting from technical problems with the glucometers. ${ }^{[13]}$

The results of this study can be compared with a similar study conducted by Hortesensus J et al. on 123 participants who concluded that the first drop of blood can be used for self-monitored glucose testing but only after washing hands. If washing hands is not possible and hands are visibly soiled or exposed to a sugar-containing product, it is acceptable to use the second drop of blood after wiping away the first drop. The study also found that external pressure (or squeezing) of the finger can lead to unreliable readings as well. ${ }^{[14]}$

In the present study, the regression was calculated to determine the effect of BG values on the bias. The $P<0.001$ value was significant which means that the bias increased as the BG values increased. The results of this study also revealed that bias did not differ in nondiabetic and diabetic patients $(P=0.167)$ and patients who received insulin or not $(P=0.25)$.

The present study was conducted on the ICU patients, where care is taken to keep a sterile and clean environment. The hands are not going to be contaminated with dirt, and the fingers are cleaned with the alcohol swab before measuring BG values. Moreover, using the first drop of blood reduces the unnecessary blood loss in patients as blood sugar values are measured hourly, 2 hourly, or 4 hourly in the ICU. Thus, we can always prefer to use the first drop of blood.

\section{Conclusions}

The present study conducted recommends the use of first drop of blood as the results have shown that there is no statistically significant difference in the BG values from the first and second drops of blood.

\section{Acknowledgment}

I would like to thank the Almighty, Ranjeet Singh Saini my father and Late Tara Saini -my mother for unconditional support, my sister Poonam Saini, and Uncle Ranbir Singh Saini, for sacrifices and support, and Abhishek Varma for extended help all the times. I owe my completion of thesis to Dr. Sukhpal Kaur and Dr. L N Yadannapudi without whom this was not possible.

\section{Financial support and sponsorship \\ Nil.}

\section{Conflicts of interest}

There are no conflicts of interest.

\section{References}

1. Clain J, Ramar K, Surani SR.Glucose control in critical care. World J Diabetes 2015;6:1082-91.

2. The Johns Hopkins Medical Institution Point-of-Care Glucose Testing Using the Nova StatStrip Glucose Hospital Meter for Current Glucometer Operators. Available from: http://www.zotero:// attachment/31/. [Last accessed on 2015 Mar 26].

3. Marx J, Hockberger R, Walls R, Adams J. Rosen's Emergency Medicine. St. Louis: Mosby; 2010. p. 3163.

4. Sarvghadi F, Yasari F. Causes and clinical presentation of hypoglycemia in patients with low blood glucose admitted to the emergency ward. Int .J Endocrinol Metab 2007;4:119. Available from: http//www.endometabol. $\mathrm{com} /$ ?page $=$ article\&article_id=1982. [Last accessed on 2015 Mar 29]

5. Alex A. Hyperglycemia (High Blood Glucose). American Diabetes Association. Available from: http://www.diabetes.org/ living-with-diabetes/treatment-and-care/blood-glucose-control/ hyperglycemia.html. [Last accessed on 2015 Mar 29].

6. Stress, Illness and High Blood Sugar - Mayo Clinic. Available from: http://www.mayoclinic.org/diseases-conditions/diabetes/expert-blog/ diabetes-blog/bgp-20056560. [Last accessed on 2015 Mar 29].

7. Tirimaceo R, Tideman PA, Dunbar J, Simpson PA, Philpot B, Laatikainen T. Should Capillary Blood Glucose Measurements be Used in Population Surveys? Science Direct. Available from: http:// www.sciencedirect.com/science/article/pii/S1877593409000599. [Last accessed on 2015 Mar 29].

8. 10 Ways to Master Your Blood Glucose Meter: Diabetes Forecast $^{\circledR}$. Available from: http://www.diabetesforecast.org/2012/ apr/10-ways-to-master-your-blood-glucose-meter.html. [Last cited on 2015 Mar 27].

9. No Coding - What is No Coding and the Importance of Coding; 2016. Available from: http://www.diabetes.co.uk/diabetes_care/ the-importance-of-coding.html. [Last cited on 2016 Oct 22].

10. $1^{\text {st }}$ ed. 2016. Available from: https://www.manualslib.com/ manual/917266/Sd-Codefree.html. [Last cited on 2016 Oct 22].

11. Kahn CR, Weir GL, King GL, Jacohson AM, Moses AC, Smith RJ. Joslin's Diabetes Melitus. 14 $4^{\text {th }}$ ed. Boston: Lippincott Williams and Wilkins; 2005. p. 1149.

12. Li M, Wang X, Shan Z. Deciding between using the first or second drop of blood for the self monitoring of blood glucose. Prim Care Diabetes 2014;8:365-9.

13. Palese A, Fabbro E, Casetta A, Mansutti I. First or second drop of blood in capillary glucose monitoring: Findings from a quantitative study. J Emerg Nurs 2016;42:420-6.

14. Hortensius J, Slingerland RJ, Kleefstra N, Logtenberg SJ, Groenier KH, Houweling ST, et al. Self-monitoring of blood glucose: The use of the first or the second drop of blood. Diabetes Care 2011;34:556-60. 